\title{
A razão áurea e os números de Fibonacci
}

\author{
Yuri Teles Moura
}

\begin{abstract}
Resumo
Neste artigo abordam-se a razão áurea e os números de Fibonacci evidenciando a relação que há entre esses entes matemáticos já tão mistificados. Apresentam-se duas construções com régua e compasso de segmentos na razão áurea. Demonstra-se que a razão entre cada termo da sequência de Fibonacci, $\mathrm{F}_{\mathrm{n}}$, e seu predecessor, $\mathrm{F}_{\mathrm{n}-1}$, é tanto mais próxima da razão áurea quanto maior o inteiro n. Prova-se a fórmula de Binet - a qual é utilizada para obter outras identidades - de duas maneiras, a saber: por meio da matriz de recorrência e a partir da equação característica. Demonstra-se a fórmula de Cassini e obtém-se, mediante o método de Newton, uma sequência de aproximações que converge rapidamente para a razão áurea.
\end{abstract}

Palavras-chave: razão áurea; sequência de Fibonacci; fórmula de Binet; fórmula de Cassini; método de Newton.

\begin{abstract}
In this article, the golden ratio and Fibonacci numbers are addressed, highlighting the relationship that exists between these already mystified mathematical entities. There are two constructions with ruler and compass of segments in the golden ratio. It is shown that the ratio between each term of the Fibonacci sequence, $\mathrm{F}_{\mathrm{n}}$, and its predecessor, $\mathrm{F}_{\mathrm{n}-1}$, is closer to the golden ratio the greater the integer n. Binet's formula - which is used to obtain other identities - is demonstrated in two ways, namely: through the recurrence matrix and from the characteristic equation. Cassini's formula is proven, and a sequence of approximations is obtained, using Newton's method, which quickly converges to the golden ratio.
\end{abstract}

Key-words: golden ratio; Fibonacci sequence; Binet's formula; Cassini's formula; Newton's method.

\section{Introdução}

Neste artigo desenvolvemos uma revisão bibliográfica cujo propósito é investigar a origem dos números de Fibonacci e da razão áurea, evidenciando a relação entre eles existente. Por acreditarmos que a matemática é feita das pessoas que se dedicam a resolver problemas, identificar padrões, compreender relações - homens e mulheres que buscam se apropriar dessa linguagem para, através dela, melhor poderem se expressar -, é que nos empenhamos em mencionar aqueles que se debruçaram sobre o tema e contribuíram para o seu desenvolvimento.

Na Seção 2, apresentaremos o contexto em que surge a razão áurea, bem como duas construções de segmentos áureos com régua e compasso. Definiremos triângulo áureo e veremos que ele aparece na construção do pentágono e decágono regulares. Definiremos também retângulo áureo, e 
mostraremos que a curva resultante da união infinita de determinados arcos circulares aproxima a espiral logarítmica.

Na Seção 3, veremos como a sequência de Fibonacci emerge da solução do problema da reprodução de coelhos, publicado no século XIII. Provaremos que, nessa sequência, a razão entre cada termo, $\mathrm{F}_{\mathrm{n}}$, e seu antecessor, $\mathrm{F}_{\mathrm{n}-1}$, é tanto mais próxima da razão áurea quanto maior é o inteiro $\mathrm{n}$. Demonstraremos a fórmula de Cassini, utilizando o princípio de indução finita, e a fórmula de Binet - que fornece $\mathrm{F}_{\mathrm{n}}$ em função de sua posição na sequência, $\mathrm{n}$-, associando a recorrência à sua equação característica e utilizando autovalores de uma matriz associada a essa sequência. Usaremos a fórmula de Binet para demonstrar algumas identidades. Por fim, obteremos uma sequência de aproximações para a raiz positiva da equação $\mathrm{x}^{2}-\mathrm{x}-1=0$ por meio do método de Newton.

Destarte, esperamos convencer o leitor da importância deste assunto, com aplicação em diversas áreas da matemática, como geometria, teoria dos números, álgebra linear, análise real etc., e que tem atraído a atenção de vários matemáticos. Nosso objetivo é apresentá-lo de forma simples e leve, além de enfatizar o caráter dinâmico dessa ciência, cujo progresso apoia-se em nossa alegria de descobrir.

\section{A razão áurea}

O termo "proporção divina" foi utilizado por Fra Luca Pacioli, em 1509, e, possivelmente mais cedo, por Pier della Francesca [1, p. 233]. Enquanto sectio divina e proportio divina aparecem nos trabalhos do matemático e astrônomo alemão Joahannes Kepler (1571-1630).

A obra de Pacioli terminou por influenciar outros autores, fazendo pairar sobre a razão áurea um certo misticismo: em um trabalho publicado em 1569, P. Ramus associou a Trindade às três partes da razão áurea. Kepler disse tratar-se de uma joia preciosa: "A geometria tem dois grandes tesouros: um é o teorema de Pitágoras, o outro, a divisão de um segmento de reta em uma proporção extrema e média; a primeira podemos comparar a uma medida de ouro, a segunda é uma joia preciosa" [1, p. 234].

No século XIX, em numerosos escritos, volumosos e pouco científicos, A. Zeising afirmou que a razão áurea domina música e arquitetura, além de ser a chave para compreender toda a morfologia. Inaugurando uma perspectiva científica, Fechner aplicou o método experimental a objetos produzidos com finalidades estéticas para concluir que o retângulo cuja razão entre os lados adjacentes é áurea possui proporções mais agradáveis [1, p. 235].

Definição 1. Um ponto $\mathrm{C}$ divide internamente um segmento $\mathrm{AB}$ na razão áurea quando sua maior parte, $\mathrm{AC}$, é tantas vezes maior do que sua menor parte, $\mathrm{CB}$, quanto o segmento todo, AB, é maior do que a maior parte, AC. Em símbolos,

$$
\frac{\mathrm{AC}}{\mathrm{CB}}=\frac{\mathrm{AB}}{\mathrm{AC}} .
$$

Como $\mathrm{AB}=\mathrm{AC}+\mathrm{CB}$, podemos escrever $\mathrm{AC} / \mathrm{CB}=1+\mathrm{CB} / \mathrm{AC}$ e, fazendo $\mathrm{AC} / \mathrm{CB}=\mathrm{x}>0$, obtemos

$$
\mathrm{x}=1+\frac{1}{\mathrm{x}}
$$

Logo, o número real x que procuramos é igual ao seu inverso somado à unidade. 
Multiplicando a Equação (1) por x, obtemos $\mathrm{x}^{2}=\mathrm{x}+1$, ou ainda

$$
\mathrm{x}^{2}-\mathrm{x}-1=0
$$

Completando o quadrado,

$$
\left(\mathrm{x}-\frac{1}{2}\right)^{2}-\frac{1}{4}-1=0,
$$

rearranjando os termos da equação e extraindo-lhes a raiz quadrada, concluímos que

$$
x=\frac{1}{2}+\frac{\sqrt{5}}{2} \quad \text { ou } \quad x=\frac{1}{2}-\frac{\sqrt{5}}{2} .
$$

Ainda hemos de nos deparar com esses números algumas vezes ao longo da nossa discussão. Assim, por economia, referir-nos-emos às raízes da Equação (2) como

$$
\alpha=\frac{1+\sqrt{5}}{2} \text { e } \beta=\frac{1-\sqrt{5}}{2} .
$$

Logo, porque positivo, $\mathrm{x}=\alpha$.

\subsection{Construção com régua e compasso}

Nos Elementos de Euclides (publicados por volta de 300 a.C.), encontram-se as seguintes proposições [1, p. 232]:

1. "Cortar uma dada linha reta de sorte que o retângulo no segmento todo e em um dos segmentos seja igual ao quadrado no segmento restante" (Livro II, Proposição 11);

2. "Cortar uma dada linha finita em proporção extrema e média" (Livro VI, Proposição 30).

Mesmo que, em termos do enunciado, essas proposições sejam equivalentes, os métodos de construção provenientes delas são bastante distintos. As evidências apontam a segunda como sendo devida a Euclides, e a primeira, aos Pitagóricos (século V a.C.).

Para encontrar o ponto $\mathrm{C}$, que divide o segmento $\mathrm{AB}$ na razão áurea, realizamos o procedimento descrito a seguir, ilustrado na Figura 1.

Primeiro, marca-se o ponto médio $\mathrm{M}$ do segmento $\mathrm{AB}$. Com a ponta seca do compasso em B e abertura AM, toma-se o ponto D sobre a reta que é perpendicular a AB e passa por B. Traça-se, então, um círculo com centro em $\mathrm{D}$ e raio $\mathrm{BD}$, que intersecta $\mathrm{AD}$ no ponto $\mathrm{E}$. Por fim, traça-se um círculo com centro em $\mathrm{A}$ e raio $\mathrm{AE}$, que intersecta $\mathrm{AB}$ no ponto $\mathrm{C}$ requerido.

De fato,

$$
\mathrm{AB}=2 \mathrm{BD}=2 \mathrm{DE}
$$

e, aplicando o teorema de Pitágoras ao triângulo ABD, retângulo em B, obtemos

$$
\mathrm{AD}^{2}=\mathrm{AB}^{2}+\mathrm{BD}^{2}=4 \mathrm{BD}^{2}+\mathrm{BD}^{2}=5 \mathrm{BD}^{2},
$$

donde, $\mathrm{AD}=\sqrt{5} \mathrm{BD}$. 


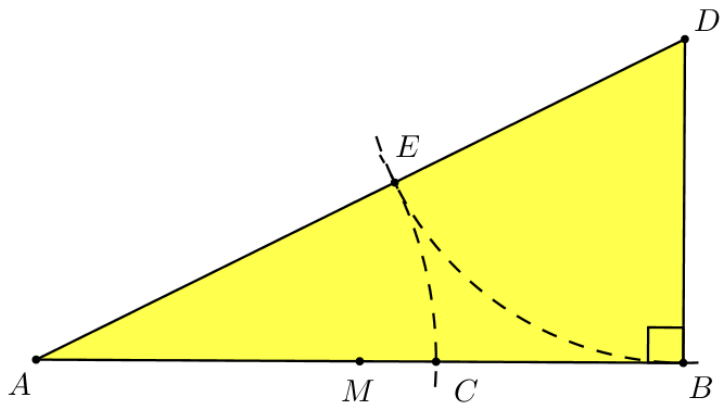

Figura 1: Construção de segmentos na razão áurea.

Daí, como DE = BD,

$$
\mathrm{AC}=\mathrm{AE}=\mathrm{AD}-\mathrm{DE}=\sqrt{5} \mathrm{BD}-\mathrm{BD} .
$$

Portanto,

$$
\frac{\mathrm{AB}}{\mathrm{AC}}=\frac{2 \mathrm{BD}}{(\sqrt{5}-1) \mathrm{BD}}=\frac{2}{\sqrt{5}-1}=\alpha .
$$

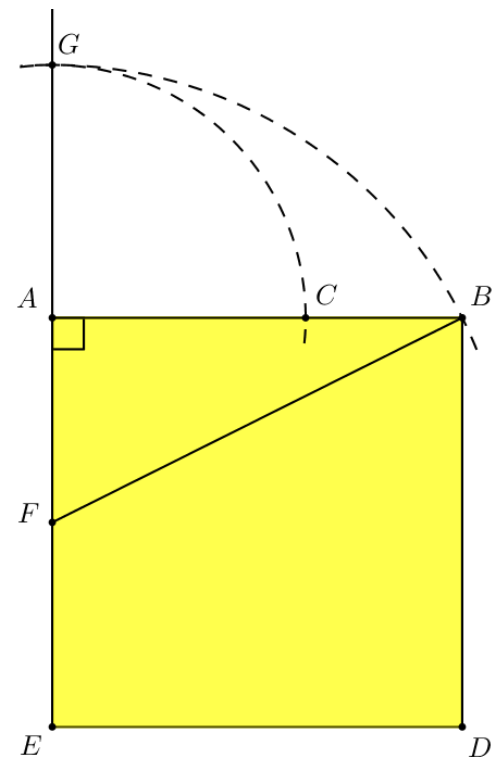

Figura 2: Outra construção de segmentos na razão áurea.

Apresentamos a seguir um outro método para construção de segmentos na razão áurea, ilustrado na Figura 2 e frequentemente atribuído ao matemático suíço Leonhard Euler (1707-1783), que o empregou em suas construções. No entanto, o método talvez já fosse conhecido pelos Pitagóricos [2, p. 244]. 
Começa-se por construir um quadrado ABDE. Marca-se o ponto médio F do lado AE e traça-se um círculo com centro em $\mathrm{F}$ e raio $\mathrm{FB}$, que intersecta a semirreta $\overrightarrow{\mathrm{FA}}$ em $\mathrm{G}$. Daí, traça-se um círculo com centro em A e raio AG, que intersecta $\mathrm{AB}$ no ponto $\mathrm{C}$.

Com efeito, uma vez que $\mathrm{AB}=2 \mathrm{AF}$, aplicamos o teorema de Pitágoras ao triângulo ABF, retângulo em A, para obter

$$
\mathrm{FB}^{2}=\mathrm{AF}^{2}+\mathrm{AB}^{2}=\mathrm{AF}^{2}+4 \mathrm{AF}^{2}=5 \mathrm{AF}^{2} .
$$

Logo, $\mathrm{FB}=\sqrt{5} \mathrm{AF}$ e, como $\mathrm{FG}=\mathrm{FB}$,

$$
\mathrm{AC}=\mathrm{FG}-\mathrm{AF}=\mathrm{FB}-\mathrm{AF}=\sqrt{5} \mathrm{AF}-\mathrm{AF} .
$$

Portanto,

$$
\frac{\mathrm{AB}}{\mathrm{AC}}=\frac{2 \mathrm{AF}}{(\sqrt{5}-1) \mathrm{AF}}=\frac{2}{\sqrt{5}-1}=\alpha .
$$

Dessa forma, aprendemos duas maneiras de dividir, com régua e compasso, segmentos na razão áurea. O leitor interessado encontrará nas referências outras duas construções de segmentos áureos $[3,4]$.

\subsection{Triângulos áureos}

Na Proposição 10 do Livro IV dos Elementos, Euclides utilizou segmentos áureos "para construir um triângulo isósceles com cada um dos ângulos da base medindo o dobro do ângulo remanescente". A partir desse triângulo, ele constrói um pentágono regular (Livro IV, Proposição 11) [1, p. 233].

Definição 2. Um triângulo isósceles é um triângulo áureo quando a razão entre um de seus lados côngruos e a base é igual a $\alpha$.

Os triângulos $\mathrm{ABC}$ e BCD da Figura 3 são áureos, e no próximo lema aprendemos a obter um triângulo áureo a partir de outro.

Lema 1. Sejam $\triangle \mathrm{ABC}$ um triângulo áureo e $\mathrm{D}$ um ponto sobre o lado $\mathrm{AC}$ que o divide na razão áurea (sendo $\mathrm{AD}>\mathrm{DC}$ ). Então $\triangle \mathrm{BCD}$ também é um triângulo áureo e $\mathrm{BD}$ bisecta $\angle \mathrm{B}$.

Demonstração. Temos que

$$
\frac{\mathrm{AB}}{\mathrm{BC}}=\frac{\mathrm{AD}}{\mathrm{CD}}=\alpha,
$$

$\mathrm{AC} / \mathrm{AD}=\alpha$ e $\mathrm{AC}=\mathrm{AB}$. Daí,

$$
\frac{\mathrm{AB}}{\mathrm{BC}}=\frac{\mathrm{BC}}{\mathrm{CD}}
$$

e, como $\angle \mathrm{ABC}=\angle \mathrm{BCD}$, são semelhantes os triângulos $\mathrm{ABC}$ e BCD pelo caso LAL de semelhança [5, p. 161]. Portanto, $\triangle \mathrm{BCD}$ também é isósceles, e $\angle \mathrm{A}=\angle \mathrm{CBD}, \angle \mathrm{ABC}=\angle \mathrm{C}=\angle \mathrm{CBD}=\angle \mathrm{BDC}$, conforme indica a Figura 3.

Isso quer dizer que $\triangle \mathrm{BCD}$ é um triângulo áureo, porque isósceles e $\mathrm{BC}=\alpha \mathrm{CD}$. Além disso, $\mathrm{AD}=\mathrm{BD}$ pois

$$
\frac{\mathrm{AD}}{\mathrm{BD}}=\frac{\mathrm{AC}-\mathrm{CD}}{\mathrm{BC}}=\frac{\mathrm{AB}}{\mathrm{BC}}-\frac{\mathrm{CD}}{\mathrm{BC}}=\alpha-\frac{1}{\alpha}=1 .
$$

Logo, $\triangle \mathrm{DAB}$ também é isósceles e, conseguintemente, $\angle \mathrm{ABD}=\angle \mathrm{A}=\angle \mathrm{DBC}$ e $\mathrm{BD}$ é bissetriz de $\angle \mathrm{B}$, pois $\angle \mathrm{ABD}=\angle \mathrm{DBC}$. 


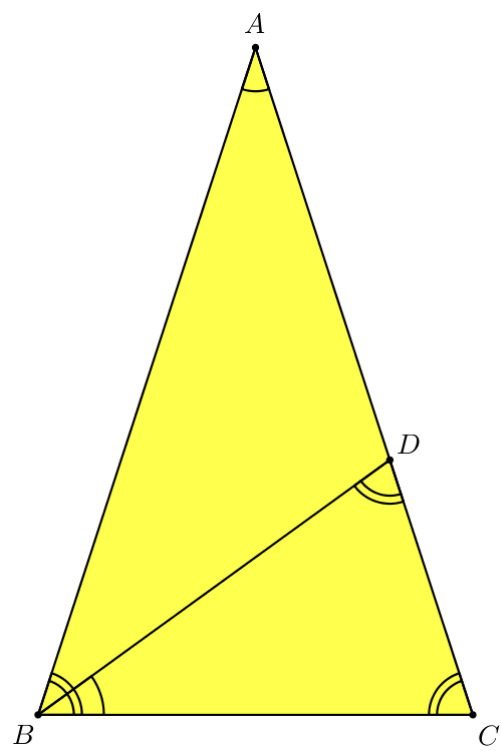

Figura 3: Triângulos áureos.

No próximo teorema estabelecemos uma condição necessária e suficiente para que um triângulo isósceles seja áureo.

Teorema 1. Um triângulo isósceles é áureo se, e somente se, seu ângulo interno não repetido é igual a $36^{\circ}$.

Demonstração. Suponhamos $\triangle \mathrm{ABC}$ áureo. Utilizando o Lema 1 , fazendo $\angle \mathrm{A}=\mathrm{x}$ e somando os ângulos internos do triângulo BCD (Figura 3), obtemos $5 \mathrm{x}=180^{\circ}$, ou seja, $\mathrm{x}=36^{\circ}$.

Por outro lado, se $\triangle \mathrm{ABC}$ é isósceles, com $\angle \mathrm{A}=36^{\circ}$, então $\angle \mathrm{B}=\angle \mathrm{C}=72^{\circ}$. Traçamos $\mathrm{AD}$, bissetriz de $\angle \mathrm{B}$. Daí, os triângulos $\mathrm{DAB}$ e $\mathrm{BCD}$ são isósceles, donde $\mathrm{BD}=\mathrm{AD}=\mathrm{BC}$ e $\triangle \mathrm{ABC}$ é semelhante a $\triangle B C D$. Portanto,

$$
\frac{\mathrm{AC}}{\mathrm{BC}}=\frac{\mathrm{BC}}{\mathrm{CD}}
$$

ou, já que $\mathrm{AC}=\mathrm{AD}+\mathrm{CD}$ e $\mathrm{AD}=\mathrm{BC}$,

$$
1+\frac{\mathrm{DC}}{\mathrm{BC}}=\frac{\mathrm{BC}}{\mathrm{CD}}
$$

donde

$$
\frac{\mathrm{BC}}{\mathrm{CD}}=\frac{\mathrm{AC}}{\mathrm{BC}}=\alpha
$$

Para ver que o triângulo $\mathrm{ABC}$, destacado no decágono regular da Figura 4a, é áureo, primeiro notamos que ele é isósceles, pois $\mathrm{AB}=\mathrm{AC}$ (raio do círculo circunscrito), depois vemos que $\angle \mathrm{A}=$ $360^{\circ} / 10=36^{\circ}$ e aplicamos o Teorema 1 . 


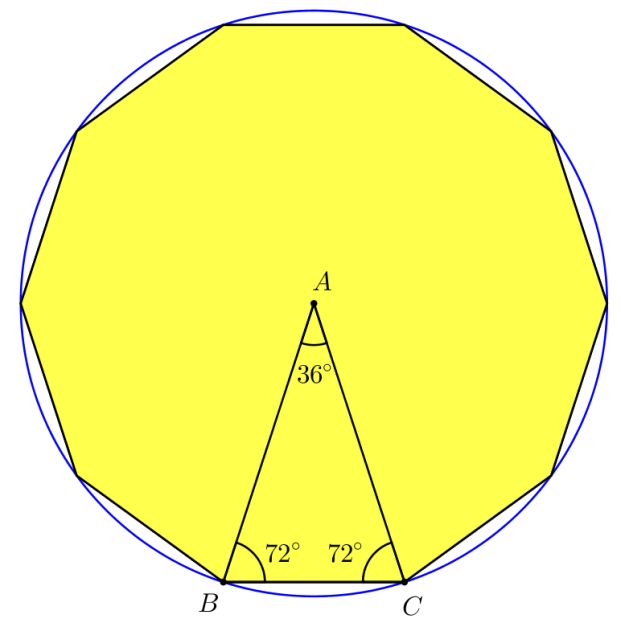

(a) Decágono regular.

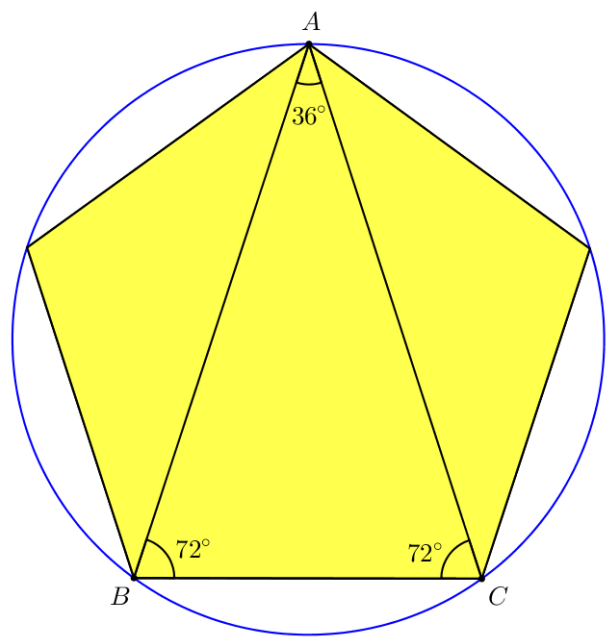

(b) Pentágono regular.

Figura 4: O triângulo áureo na construção de dois polígonos regulares.

Quanto àquele da Figura 4b: cada ângulo interno do pentágono mede $(5-2) 180^{\circ} / 5=108^{\circ}$; cordas congruentes (os lados do pentágono regular opostos ao vértice A) determinam ângulos sobre o círculo também congruentes [5, pp. 107-109]. Logo, $\angle \mathrm{BAC}=108^{\circ} / 3=36^{\circ}$, e como as diagonais $\mathrm{AB}$ e $\mathrm{AC}$ do pentágono são congruentes, concluímos do Teorema 1 que $\triangle \mathrm{ABC}$ é áureo.

\subsection{Retângulos áureos}

Na Sub-seção 2.1 vimos que a Proposição 11 do Livro II dos Elementos de Euclides trata da divisão de um segmento de reta tal que "o retângulo no segmento todo e em um dos segmentos seja igual ao quadrado no segmento restante".

Sejam a e b as partes do segmento que resultam dessa divisão. Com a igualdade do retângulo e do quadrado, isto é, de suas áreas, temos que $(a+b) b=a^{2}$, ou ainda, $a b+b^{2}=a^{2}$. Dividindo essa equação por $\mathrm{b}^{2}$ e fazendo $\mathrm{x}=\frac{\mathrm{a}}{\mathrm{b}}$, obtemos $\mathrm{x}+1=\mathrm{x}^{2}$, cujas raízes são $\alpha$ e $\beta$.

Definição 3. Denominamos áureo todo retângulo cuja razão entre os lados maior e menor é igual à razão áurea.

$\mathrm{O}$ retângulo $\mathrm{ABCD}$ da Figura 5, construído de sorte que $\mathrm{AB}=\alpha \mathrm{BC}$, é áureo, ao passo que o retângulo BCEF é áureo sempre que DAFE for um quadrado. Reciprocamente, DAFE é um quadrado sempre que BCEF for um retângulo áureo.

Teorema 2. Seja k > 1 a razão entre o comprimento e a largura em um retângulo $\mathrm{ABCD}$, do qual é removido o retângulo $\mathrm{BCEF}$ semelhante ao primeiro. Nessas condições, a razão entre as áreas dos retângulos original, $\mathrm{ABCD}$, e remanescente, DAFE, é $\mathrm{k}$ se, e somente se, $\mathrm{k}=\alpha e$, consequentemente, DAFE é um quadrado.

Demonstração. Temos que $\mathrm{k}>1$ é a razão entre o comprimento $\mathrm{AB}$ e a largura BC do retângulo 


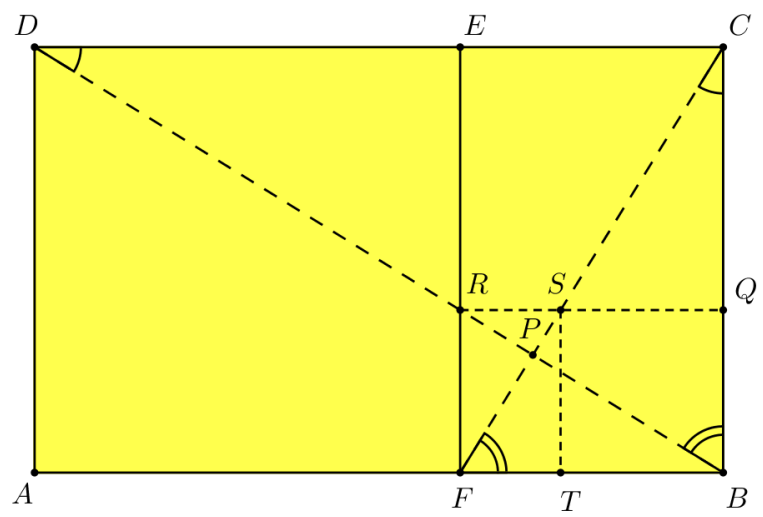

Figura 5: Retângulos áureos.

$\mathrm{ABCD}$, isto é, $\mathrm{AB} / \mathrm{BC}=\mathrm{k}$. Além disso, os retângulos $\mathrm{ABCD}$ e BCEF são semelhantes, ou seja,

$$
\mathrm{k}=\frac{\mathrm{AB}}{\mathrm{BC}}=\frac{\mathrm{BC}}{\mathrm{FB}}
$$

Suponhamos que a razão entre as áreas dos retângulos ABCD e DAFE seja igual a k:

$$
\frac{\mathrm{AB} \cdot \mathrm{BC}}{\mathrm{AF} \cdot \mathrm{FE}}=\mathrm{k} \text {. }
$$

Daí, como $\mathrm{FE}=\mathrm{BC}$ e $\mathrm{FB}=\frac{1}{\mathrm{k}} \mathrm{BC}$,

$$
\begin{aligned}
\mathrm{k} & =\frac{\mathrm{AB} \cdot \mathrm{BC}}{\mathrm{AF} \cdot \mathrm{BC}}=\frac{\mathrm{AB}}{\mathrm{AF}}=\frac{\mathrm{AB}}{\mathrm{AB}-\mathrm{FB}} \\
& =\frac{1}{1-\frac{\mathrm{FB}}{\mathrm{AB}}}=\frac{1}{1-\frac{1}{\mathrm{k}} \frac{\mathrm{BC}}{\mathrm{AB}}}=\frac{1}{1-\frac{1}{\mathrm{k}^{2}}},
\end{aligned}
$$

isto é, $1 / \mathrm{k}=1-1 / \mathrm{k}^{2}$. Multiplicando esta equação por $\mathrm{k}^{2}$ obtemos $\mathrm{k}=\mathrm{k}^{2}-1$, que é igual à Equação (2), apenas a variável tem nome diferente. Logo, $\mathrm{k}=\alpha$ e, portanto,

$$
\frac{\mathrm{AF}}{\mathrm{FE}}=\frac{\mathrm{AB}-\mathrm{FB}}{\mathrm{BC}}=\frac{\mathrm{AB}}{\mathrm{BC}}-\frac{\mathrm{FB}}{\mathrm{BC}}=\alpha-\frac{1}{\alpha}=1,
$$

e DAFE é um quadrado.

Suponhamos agora $\mathrm{k}=\alpha$. Daí,

$$
\frac{\mathrm{AB} \cdot \mathrm{BC}}{\mathrm{AF} \cdot \mathrm{FE}}=\frac{1}{1-\frac{1}{\alpha^{2}}}=\alpha
$$

Definição 4. O recíproco de um retângulo é outro menor, semelhante ao primeiro, tendo com ele um lado comum. 
Na Figura 5, o retângulo BCEF é recíproco de ABCD. O quadrado DAFE é a menor figura que quando adicionada ao retângulo BCEF resulta em um retângulo semelhante ao primeiro [2, p. 288].

Teorema 3. As diagonais de dois retângulos recíprocos são perpendiculares entre si.

Demonstração. Seja $\mathrm{P}$ a intersecção das diagonais $\mathrm{BD}$ e $\mathrm{CF}$ dos retângulos recíprocos $\mathrm{ABCD}$ e BCEF, como na Figura 5.

Posto que

$$
\frac{\mathrm{CD}}{\mathrm{BC}}=\frac{\mathrm{BC}}{\mathrm{BF}}=\alpha,
$$

os triângulos retângulos $\mathrm{BCD}$ e FBC são semelhantes. Logo, $\angle \mathrm{CBD}=\angle \mathrm{BFC}$ e $\angle \mathrm{BDC}=\angle \mathrm{BCF}$.

Assim, $\angle \mathrm{PBC}+\angle \mathrm{PCB}=90^{\circ} \mathrm{e}$, portanto, $\angle \mathrm{BPC}=90^{\circ}$, ou seja, BD é perpendicular a $\mathrm{CF}$.

O Teorema 3 provê uma maneira sistemática de construir retângulos recíprocos. Com efeito, partindo dos retângulos recíprocos $\mathrm{ABCD}$ e BCEF da Figura 5, obtemos o retângulo recíproco de $\mathrm{BCEF}$, assim: tomamos $\mathrm{R}$ na intersecção de BD com EF, e traçamos QR perpendicular a BC. Tomar R sobre BD faz que BR seja perpendicular a CF, já que BD e CF são perpendiculares, e, do Teorema 3, BQRF é o retângulo recíproco de BCEF.

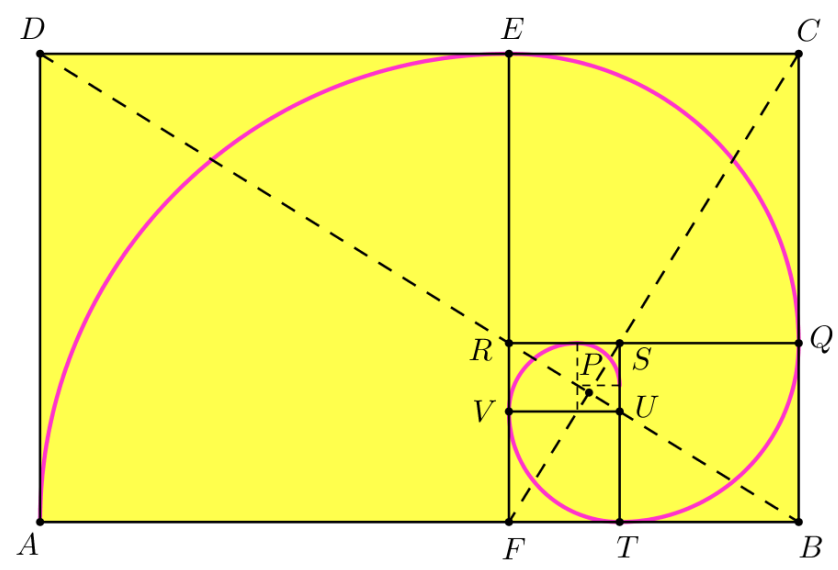

Figura 6: Espiral de arcos circulares.

De maneira análoga, podemos desenhar também o retângulo áureo recíproco de BQRF. De fato, sendo S a intersecção de CF com QR, traçamos ST perpendicular a BF, e o Teorema 3 garante-nos que FTSR é o retângulo (áureo) recíproco de BQRF.

Continuando esse procedimento indefinidamente, produzir-se-á uma sequência de retângulos áureos cada vez menores, como ilustra a Figura 6, que também destaca a curva obtida da reunião dos sucessivos arcos circulares de raios iguais aos lados dos quadrados, DAFE, CERQ, BQST, TUVF etc. Essa curva tangencia cada um dos retângulos áureos em pontos que os dividem na razão áurea. Esses pontos espiralam em direção a P, interseção das diagonais dos retângulos originais, e estão sobre a espiral logarítmica, com polo em P. 
Há uma sequência de infinitos pontos ..., V, T, Q, E, A, ... cujas coordenadas polares

$$
\mathrm{r}=\alpha^{\mathrm{n}}, \quad \theta=\frac{\pi}{2} \mathrm{n}
$$

satisfazem a equação $\mathrm{r}=\alpha^{\frac{2 \theta}{\pi}}$. Logo, todos esses pontos estão sobre a espiral logarítmica

$$
\mathrm{r}=\mu^{\theta}
$$

onde $\mu=\alpha^{\frac{2}{\pi}}$. Essa espiral é apenas aproximada pela curva da Figura 6, pois a espiral logarítmica intersecta os lados dos quadrados em ângulos bem pequenos, em vez de tangenciá-los [6, p. 165].

\section{Os números de Fibonacci}

Leonardo Pisano (Figura 7) viveu entre 1170 e 1250 e foi reponsável por introduzir na Europa o sistema de numeração hindu-arábico que usamos hoje. Em seu Liber Abaci, publicado em 1202, Fibonacci (filho de Bonacci), como ficou conhecido, apresentou o problema sobre reprodução de coelhos, que está relacionado à sua célebre sequência de números inteiros $\mathrm{F}_{\mathrm{n}}$.

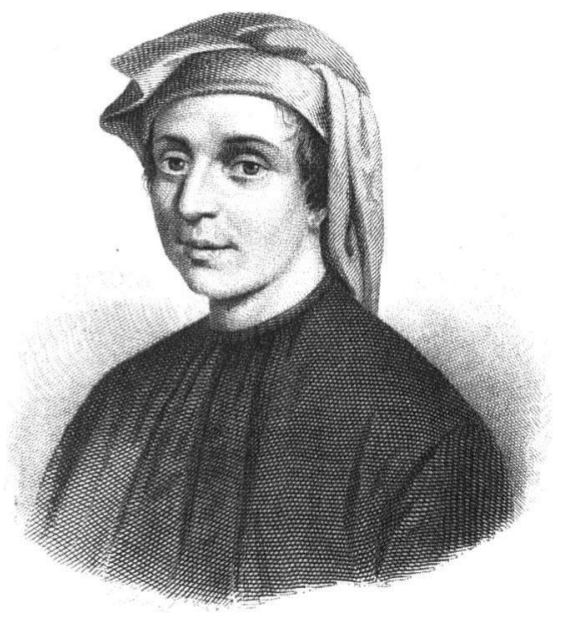

Figura 7: Leonardo Pisano [2, p. 2].

Nesse problema, Fibonacci assumiu que os coelhos viveriam eternamente e que, a cada mês, um par de coelhos dá origem a um novo par, o qual se torna produtivo a partir da idade de dois meses. No primeiro mês, o experimento começa com um par de coelhos recém-nascidos. Assim, no segundo mês ainda há apenas um par. No terceiro mês há 2 ; no quarto, 3 ; no quinto, 5 ; e assim por diante. Seja $\mathrm{F}_{\mathrm{n}}$ o número de pares de coelhos no n-ésimo mês.

Quatro séculos mais tarde, em 1611, Kepler escreveu de forma explícita o que Fibonacci não pode ter deixado de notar: cada termo $\mathrm{F}_{\mathrm{n}}$, para $\mathrm{n} \geq 2$, é obtido da soma dos dois termos antecedentes [6, p. 166], porquanto a sequência fica determinada pela fórmula recursiva

$$
\mathrm{F}_{\mathrm{n}}=\mathrm{F}_{\mathrm{n}-1}+\mathrm{F}_{\mathrm{n}-2} \text {, }
$$

e pelas condições iniciais $\mathrm{F}_{0}=0$ e $\mathrm{F}_{1}=1$. 
Tabela 1: Os números de Fibonacci

\begin{tabular}{ccccccccccccc}
$\mathrm{n}$ & 0 & 1 & 2 & 3 & 4 & 5 & 6 & 7 & 8 & 9 & 10 & $\cdots$ \\
$\mathrm{F}_{\mathrm{n}}$ & 0 & 1 & 1 & 2 & 3 & 5 & 8 & 13 & 21 & 34 & 55 & $\cdots$ \\
$\mathrm{F}_{\mathrm{n}+1} / \mathrm{F}_{\mathrm{n}}$ & $\infty$ & 1 & 2 & 1,5 & 1,6667 & 1,6 & 1,625 & 1,6154 & 1,6190 & 1,6176 & 1,6182 & $\cdots$ \\
\hline
\end{tabular}

Kepler observou também que, quanto maior é o inteiro $n$, mais as razões $F_{n+1} / F_{n}$ aproximam-se do número

$$
\alpha=\frac{\sqrt{5}+1}{2} \approx 1,618034,
$$

como indicam os dados na terceira linha da Tabela 1. Outros cem anos passaram-se antes que R. Simson (1687-1768) utilizasse a relação

$$
\alpha=1+\frac{1}{\alpha}
$$

para mostrar que a sequência das frações

$$
\begin{gathered}
1+\frac{1}{1}=\frac{2}{1}=\frac{\mathrm{F}_{3}}{\mathrm{~F}_{2}}, \\
1+\frac{1}{1+\frac{1}{1}}=\frac{3}{2}=\frac{\mathrm{F}_{4}}{\mathrm{~F}_{3}}, \\
1+\frac{1}{1+\frac{1}{1+\frac{1}{1}}}=\frac{5}{3}=\frac{\mathrm{F}_{5}}{\mathrm{~F}_{4}}
\end{gathered}
$$

etc., converge para $\alpha$.

Com efeito, dividindo a recorrência, $F_{n+1}=F_{n}+F_{n-1}$, da sequência de Fibonacci, por $F_{n}$, obtemos

$$
\frac{F_{n+1}}{F_{n}}=1+\frac{F_{n-1}}{F_{n}} .
$$

Fazendo

$$
\lim _{n \rightarrow \infty} \frac{F_{n+1}}{F_{n}}=\lim _{n \rightarrow \infty} \frac{F_{n}}{F_{n-1}}=x,
$$

vemos que $\mathrm{x}=1+1 / \mathrm{x}$, ou seja, $\mathrm{x}=\alpha$.

O matemático francês Édouard Lucas (Figura 8) propôs diferentes condições iniciais para a sequência de Fibonacci. Os números $\mathrm{L}_{\mathrm{n}}$, definidos pela fórmula de recorrência

$$
\mathrm{L}_{\mathrm{n}}=\mathrm{F}_{\mathrm{n}-1}+\mathrm{F}_{\mathrm{n}+1}, \quad \mathrm{n} \geq 2,
$$

com $\mathrm{L}_{0}=2$ e $\mathrm{L}_{1}=1$, são chamados números de Lucas, em sua homenagem.

Vemos que $\mathrm{L}_{2}=\mathrm{F}_{1}+\mathrm{F}_{3}=1+2=3, \mathrm{~L}_{3}=\mathrm{F}_{2}+\mathrm{F}_{4}=1+3=4$, e assim por diante.

Esses números satisfazem à relação

$$
\mathrm{L}_{\mathrm{n}}=\mathrm{L}_{\mathrm{n}-1}+\mathrm{L}_{\mathrm{n}-2}, \quad \mathrm{n} \geq 3,
$$

como podemos verificar por indução. 


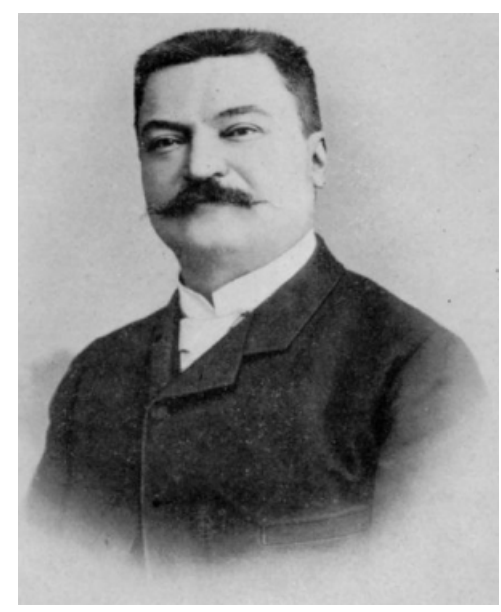

Figura 8: François-Édouard-Anatole Lucas (1842-1891).

Tabela 2: Os números de Lucas

\begin{tabular}{ccccccccccccc}
$\mathrm{n}$ & 0 & 1 & 2 & 3 & 4 & 5 & 6 & 7 & 8 & 9 & 10 & $\cdots$ \\
$\mathrm{L}_{\mathrm{n}}$ & 2 & 1 & 3 & 4 & 7 & 11 & 18 & 29 & 47 & 76 & 123 & $\cdots$ \\
$\mathrm{L}_{\mathrm{n}+1} / \mathrm{L}_{\mathrm{n}}$ & 0,5 & 3 & 1,3333 & 1,75 & 1,57 & 1,636 & 1,611 & 1,621 & 1,617 & 1,6184 & 1,6179 & $\cdots$ \\
\hline
\end{tabular}

De fato,

$$
\begin{aligned}
\mathrm{L}_{\mathrm{n}+1} & =\mathrm{F}_{\mathrm{n}}+\mathrm{F}_{\mathrm{n}+2}=\mathrm{F}_{\mathrm{n}-1}+\mathrm{F}_{\mathrm{n}-2}+\mathrm{F}_{\mathrm{n}+1}+\mathrm{F}_{\mathrm{n}} \\
& =\mathrm{F}_{\mathrm{n}+1}+\mathrm{F}_{\mathrm{n}-1}+\mathrm{F}_{\mathrm{n}}+\mathrm{F}_{\mathrm{n}-2}=\mathrm{L}_{\mathrm{n}}+\mathrm{L}_{\mathrm{n}-1} .
\end{aligned}
$$

Logo, a sequência de Lucas é um tipo particular da de Fibonacci, e $\lim _{n \rightarrow \infty} L_{n+1} / L_{n}=\alpha$, como indicam os dados da Tabela 2.

\subsection{Algumas identidades}

A identidade no teorema abaixo, cuja prova faremos por indução, foi descoberta, em 1680, pelo matemático e astrônomo francês, nascido na Itália, Giovanni Domenico Cassini (1625-1712). Robert Simson (1687-1768), da Universidade de Glasgow, também a descobriu, de forma independente, em 1753 [2, pp. 74-75].

Teorema 4 (Cassini). Seja n um inteiro $\geq 1$. Então,

$$
\mathrm{F}_{\mathrm{n}-1} \mathrm{~F}_{\mathrm{n}+1}-\mathrm{F}_{\mathrm{n}}^{2}=(-1)^{\mathrm{n}}
$$

Demonstração. Uma vez que $\mathrm{F}_{0} \mathrm{~F}_{2}-\mathrm{F}_{1}^{2}=0 \cdot 1-1=-1=(-1)^{1}$, a afirmação é válida para $\mathrm{n}=1$. 
Assumindo que a fórmula funcione para um inteiro $n>1$ arbitrário,

$$
\begin{aligned}
\mathrm{F}_{\mathrm{n}} \mathrm{F}_{\mathrm{n}+2}-\mathrm{F}_{\mathrm{n}+1}^{2} & =\left(\mathrm{F}_{\mathrm{n}+1}-\mathrm{F}_{\mathrm{n}-1}\right)\left(\mathrm{F}_{\mathrm{n}}+\mathrm{F}_{\mathrm{n}+1}\right)-\mathrm{F}_{\mathrm{n}+1}^{2} \\
& =\mathrm{F}_{\mathrm{n}+1} \mathrm{~F}_{\mathrm{n}}+\mathrm{F}_{\mathrm{n}+1}^{2}-\mathrm{F}_{\mathrm{n}-1} \mathrm{~F}_{\mathrm{n}}-\mathrm{F}_{\mathrm{n}-1} \mathrm{~F}_{\mathrm{n}+1}-\mathrm{F}_{\mathrm{n}+1}^{2} \\
& =\mathrm{F}_{\mathrm{n}+1} \mathrm{~F}_{\mathrm{n}}-\mathrm{F}_{\mathrm{n}-1} \mathrm{~F}_{\mathrm{n}}-\mathrm{F}_{\mathrm{n}-1} \mathrm{~F}_{\mathrm{n}+1} \\
& =\mathrm{F}_{\mathrm{n}+1} \mathrm{~F}_{\mathrm{n}}-\mathrm{F}_{\mathrm{n}-1} \mathrm{~F}_{\mathrm{n}}-(-1)^{\mathrm{n}}-\mathrm{F}_{\mathrm{n}}^{2} \\
& =\mathrm{F}_{\mathrm{n}+1} \mathrm{~F}_{\mathrm{n}}-\mathrm{F}_{\mathrm{n}}\left(\mathrm{F}_{\mathrm{n}-1}+\mathrm{F}_{\mathrm{n}}\right)-(-1)^{\mathrm{n}} \\
& =\mathrm{F}_{\mathrm{n}+1} \mathrm{~F}_{\mathrm{n}}-\mathrm{F}_{\mathrm{n}} \mathrm{F}_{\mathrm{n}+1}-(-1)^{\mathrm{n}} \\
& =(-1)^{\mathrm{n}+1},
\end{aligned}
$$

e a fórmula funciona para $n+1$. Logo, pelo princípio de indução, a afirmação é válida para todo inteiro $n \geq 1$.

Embora a fórmula de Cassini, Teorema 4, possa ser inferida de uma passagem citada por Kepler, ela é utilizada mais explicitamente por R. Simson (1753). Foi a ela também, e, portanto, à sequência de Fibonacci, que Schlegel chegou, quando procurou generalizar o conhecido paradoxo geométrico de dividir um quadrado $8 \times 8$ em quatro partes que se encaixam no retângulo $5 \times 13-$ o leitor interessado encontrará mais sobre o referido paradoxo e sua generalização nas referências [2, pp. 100-106].

No próximo corolário utilizamos a notação $(\mathrm{a}, \mathrm{b})$ para representar o maior divisor comum aos inteiros a e b.

Corolário 1. Quaisquer dois números de Fibonacci consecutivos são relativa e mutuamente primos; isto é, $\left(\mathrm{F}_{\mathrm{n}+1}, \mathrm{~F}_{\mathrm{n}}\right)=1$, para todo $\mathrm{n}$ inteiro.

Demonstração. Suponhamos n par (para n ímpar o raciocínio é análogo). Do Teorema 4,

$$
\mathrm{F}_{\mathrm{n}+1}\left(\mathrm{~F}_{\mathrm{n}-1}\right)+\mathrm{F}_{\mathrm{n}}\left(-\mathrm{F}_{\mathrm{n}}\right)=1
$$

ou seja, temos 1 como uma combinação linear dos números $\mathrm{F}_{\mathrm{n}+1}$ e $\mathrm{F}_{\mathrm{n}}$. Isto implica que $\left(\mathrm{F}_{\mathrm{n}+1}, \mathrm{~F}_{\mathrm{n}}\right)$ divide $1\left[7\right.$, p. 19]. Logo, $\left(\mathrm{F}_{\mathrm{n}+1}, \mathrm{~F}_{\mathrm{n}}\right)=1$.

Tratamos agora de obter um resultado que nos permitirá resolver recorrências lineares de segunda ordem homogêneas, com coeficientes constantes, da forma $\mathrm{x}_{\mathrm{n}+2}+\mathrm{px}_{\mathrm{n}+1}+\mathrm{qx}_{\mathrm{n}}=0$, particularmente a Recorrência (3), da sequência de Fibonacci. A cada uma dessas recorrências, associamos sua equação característica, $\mathrm{r}^{2}+\mathrm{pr}+\mathrm{q}=0[8, \mathrm{pp} .67-69]$. Podemos supor $\mathrm{q} \neq 0$, caso contrário a recorrência é de primeira ordem.

Lema 2. Se $\mathrm{r}_{1}$ e $\mathrm{r}_{2}$ são as raízes da equação $\mathrm{r}^{2}+\mathrm{pr}+\mathrm{q}=0$, então, qualquer sequência da forma $\mathrm{a}_{\mathrm{n}}=\mathrm{C}_{1} \mathrm{r}_{1}^{\mathrm{n}}+\mathrm{C}_{2} \mathrm{r}_{2}^{\mathrm{n}}$ é solução da recorrência $\mathrm{x}_{\mathrm{n}+2}+\mathrm{px}_{\mathrm{n}+1}+\mathrm{qx}_{\mathrm{n}}=0$, quaisquer que sejam as constantes $\mathrm{C}_{1} e \mathrm{C}_{2}$.

Demonstração. Substituindo $\mathrm{a}_{\mathrm{n}}=\mathrm{C}_{1} \mathrm{r}_{1}^{\mathrm{n}}+\mathrm{C}_{2} \mathrm{r}_{2}^{\mathrm{n}}$ na recorrência, obtemos, agrupando convenientemente os termos,

$$
\mathrm{a}_{\mathrm{n}+2}+\mathrm{pa}_{\mathrm{n}+1}+\mathrm{qa} \mathrm{a}_{\mathrm{n}}=\mathrm{C}_{1} \mathrm{r}_{1}^{\mathrm{n}}\left(\mathrm{r}_{1}^{2}+\mathrm{pr}_{1}+\mathrm{q}\right)+\mathrm{C}_{2} \mathrm{r}_{2}^{\mathrm{n}}\left(\mathrm{r}_{2}^{2}+\mathrm{pr}_{2}+\mathrm{q}\right)=0
$$

pois $\mathrm{r}_{1}^{2}+\mathrm{pr}_{1}+\mathrm{q}=\mathrm{r}_{2}^{2}+\mathrm{pr}_{2}+\mathrm{q}=0$. 
Teorema 5 (Binet). Se $\alpha$ é a raiz positiva da equação $\mathrm{x}^{2}-\mathrm{x}-1=0$ e $\beta$ sua raiz negativa, então

$$
\mathrm{F}_{\mathrm{n}}=\frac{\alpha^{\mathrm{n}}-\beta^{\mathrm{n}}}{\alpha-\beta} .
$$

Demonstração. À recorrência linear de segunda ordem homogênea, com coeficientes constantes, $\mathrm{F}_{\mathrm{n}+2}=\mathrm{F}_{\mathrm{n}+1}+\mathrm{F}_{\mathrm{n}}$, associamos a equação característica $\mathrm{r}^{2}=\mathrm{r}+1$, cujas raízes, já sabemos bem, são iguais a $\alpha$ e $\beta$. Aplicando o Lema 2, obtemos

$$
\mathrm{F}_{\mathrm{n}}=\mathrm{C}_{1} \alpha^{\mathrm{n}}+\mathrm{C}_{2} \beta^{\mathrm{n}}
$$

Para determinar $\mathrm{C}_{1}$ e $\mathrm{C}_{2}$, utilizamos $\mathrm{F}_{0}=0$ e $\mathrm{F}_{1}=1$ e obtemos o sistema linear

$$
\left[\begin{array}{cc}
\alpha & \beta \\
1 & 1
\end{array}\right]\left[\begin{array}{l}
\mathrm{C}_{1} \\
\mathrm{C}_{2}
\end{array}\right]=\left[\begin{array}{l}
1 \\
0
\end{array}\right],
$$

cuja solução é

$$
\mathrm{C}_{1}=-\mathrm{C}_{2}=\frac{1}{\alpha-\beta},
$$

a qual obtivemos multiplicando o sistema pela matriz

$$
\left[\begin{array}{cc}
1 & 0 \\
1 & -\alpha
\end{array}\right]
$$

Portanto,

$$
\mathrm{F}_{\mathrm{n}}=\frac{\alpha^{\mathrm{n}}-\beta^{\mathrm{n}}}{\alpha-\beta} .
$$

De maneira análoga, conseguimos uma relação explícita $\mathrm{L}_{\mathrm{n}}$.

De fato, a sequência de Lucas é um tipo particular da de Fibonacci, e pode ser representada pela recorrência $\mathrm{L}_{\mathrm{n}+2}=\mathrm{L}_{\mathrm{n}+1}+\mathrm{L}_{\mathrm{n}}$, donde

$$
\mathrm{L}_{\mathrm{n}}=\mathrm{C}_{1} \alpha^{\mathrm{n}}+\mathrm{C}_{2} \beta^{\mathrm{n}}
$$

Para determinar $\mathrm{C}_{1}$ e $\mathrm{C}_{2}$ utilizamos $\mathrm{L}_{0}=2$ e $\mathrm{L}_{1}=1$, obtendo o sistema linear

$$
\left[\begin{array}{ll}
\alpha & \beta \\
1 & 1
\end{array}\right]\left[\begin{array}{l}
\mathrm{C}_{1} \\
\mathrm{C}_{2}
\end{array}\right]=\left[\begin{array}{l}
1 \\
2
\end{array}\right] .
$$

Sua solução é $\mathrm{C}_{1}=\mathrm{C}_{2}=1$, logo

$$
\mathrm{L}_{\mathrm{n}}=\alpha^{\mathrm{n}}+\beta^{\mathrm{n}} .
$$

Outra maneira de provar o Teorema 5 consiste em utilizar um expediente da álgebra linear [9, pp. 301-302].

Para esta abordagem, lembremos que um autovalor, $\lambda$, de uma matriz quadrada A é tal que $\mathrm{Ax}=\lambda \mathbf{x}$, para um $\mathbf{x}$ conveniente $-\mathrm{o}$ vetor $\mathbf{x}$ é chamado um autovetor de $\mathrm{A}$.

Assim, podemos escrever $(A-\lambda I) \mathbf{x}=0$, e essa equação tem solução não nula sempre que

$$
\operatorname{det}(\mathrm{A}-\lambda \mathrm{I})=0
$$


(equação característica de A).

Se $\mathrm{S}$ é a matriz em cujas colunas dispõem-se os autovetores de $\mathrm{A}$ e $\Lambda$, a matriz diagonal em que estão dispostos os autovalores de $\mathrm{A}$, então $\mathrm{A}=\mathrm{S} \Lambda \mathrm{S}^{-1}$, donde $\mathrm{A}^{\mathrm{n}}=\left(\mathrm{S} \Lambda \mathrm{S}^{-1}\right)\left(\mathrm{S} \Lambda \mathrm{S}^{-1}\right) \cdots\left(\mathrm{S} \Lambda \mathrm{S}^{-1}\right)$, com n parcelas iguais a $\mathrm{A}=\mathrm{S} \Lambda \mathrm{S}^{-1}$. Daí, usando a propriedade associativa do produto de matrizes, $\mathrm{A}^{\mathrm{n}}=\mathrm{S} \Lambda\left(\mathrm{S}^{-1} \mathrm{~S}\right) \Lambda \mathrm{S}^{-1} \cdots \mathrm{S} \Lambda \mathrm{S}^{-1}=\mathrm{S} \Lambda^{\mathrm{n}} \mathrm{S}^{-1}$, pois $\mathrm{S}^{-1} \mathrm{~S}=\mathrm{I}$, a matriz identidade.

Considere a sequência $\left\{\mathbf{u}_{\mathbf{n}}\right\}$ de vetores

$$
\mathbf{u}_{0}, \quad \mathbf{u}_{1}=A \mathbf{u}_{0}, \quad \mathbf{u}_{2}=A \mathbf{u}_{1}=A^{2} \mathbf{u}_{0}, \quad \ldots, \quad \mathbf{u}_{n}=A^{\mathrm{n}} \mathbf{u}_{0}, \quad \ldots,
$$

em que cada termo é obtido multiplicando-se a matriz A pelo termo antecedente, isto é,

$$
\mathbf{u}_{\mathrm{k}+1}=\mathrm{A} \mathbf{u}_{\mathrm{k}}
$$

com $\mathrm{k}$ inteiro $\geq 0$.

Escrevendo $\mathbf{u}_{\mathbf{0}}$ como uma combinação linear dos autovetores de $\mathrm{A}$, ou seja, $\mathbf{u}_{\mathbf{0}}=\mathrm{Sc}$, onde $\mathbf{c}$ é o vetor contendo os coeficientes dessa combinação linear, obtemos $\mathbf{u}_{\mathbf{n}}=\mathrm{S} \Lambda^{\mathrm{n}} \mathbf{c}$.

A chave aqui é começar com a Equação (9) (matricial). Esta é uma recorrência linear de primeira ordem para vetores, enquanto a Equação (3), da sequência de Fibonacci, é uma recorrência linear de segunda ordem para escalares. Relacionamos essas recorrências pondo dois números de Fibonacci em um vetor:

$$
\mathbf{u}_{\mathrm{k}}=\left[\begin{array}{c}
\mathrm{F}_{\mathrm{k}+1} \\
\mathrm{~F}_{\mathrm{k}}
\end{array}\right]
$$

de modo que $\mathbf{u}_{0}=\left[\begin{array}{l}\mathrm{F}_{1} \\ \mathrm{~F}_{0}\end{array}\right]=\left[\begin{array}{l}1 \\ 0\end{array}\right]$.

A regra $F_{k+2}=F_{k+1}+F_{k}$ é escrita assim:

$$
\mathbf{u}_{\mathrm{k}+1}=\left[\begin{array}{ll}
1 & 1 \\
1 & 0
\end{array}\right] \mathbf{u}_{\mathbf{k}}
$$

Multiplica-se por $\mathrm{A}=\left[\begin{array}{ll}1 & 1 \\ 1 & 0\end{array}\right]$ em cada passo, e após $\mathrm{n}$ passos obtemos $\mathbf{u}_{\mathbf{n}}=\mathrm{A}^{\mathrm{n}} \mathbf{u}_{\mathbf{0}}$ :

$$
\mathbf{u}_{0}=\left[\begin{array}{l}
1 \\
0
\end{array}\right], \quad \mathbf{u}_{1}=\left[\begin{array}{l}
1 \\
1
\end{array}\right], \quad \mathbf{u}_{2}=\left[\begin{array}{l}
2 \\
1
\end{array}\right], \quad \mathbf{u}_{3}=\left[\begin{array}{l}
3 \\
2
\end{array}\right], \quad \ldots, \quad \mathbf{u}_{\mathbf{n}}=\left[\begin{array}{c}
F_{n+1} \\
F_{n}
\end{array}\right], \quad \ldots
$$

Subtraindo $\lambda$ da diagonal de A,

$$
A-\lambda I=\left[\begin{array}{cc}
1-\lambda & 1 \\
1 & -\lambda
\end{array}\right]
$$

de modo que

$$
\operatorname{det}(\mathrm{A}-\lambda \mathrm{I})=\lambda^{2}-\lambda-1
$$

A equação $\lambda^{2}-\lambda-1=0$, que já resolvemos na Seção 2 , tem raízes iguais a

$$
\alpha=\frac{1+\sqrt{5}}{2} \quad \text { e } \beta=\frac{1-\sqrt{5}}{2} .
$$

Esses autovalores levam a autovetores $\mathbf{x}_{1}=(\alpha, 1)$ e $\mathbf{x}_{\mathbf{2}}=(\beta, 1)$. 
Encontramos então a combinação linear desses vetores que fornece $\mathbf{u}_{0}=(1,0)$, isto é,

$$
\left[\begin{array}{l}
1 \\
0
\end{array}\right]=\frac{1}{\alpha-\beta}\left(\left[\begin{array}{l}
\alpha \\
1
\end{array}\right]-\left[\begin{array}{l}
\beta \\
1
\end{array}\right]\right),
$$

e multiplicamos $\mathbf{u}_{\mathbf{0}}$ por $\mathrm{A}^{\mathrm{n}}$ para obter $\mathbf{u}_{\mathbf{n}}$ :

$$
\left[\begin{array}{c}
\mathrm{F}_{\mathrm{n}+1} \\
\mathrm{~F}_{\mathrm{n}}
\end{array}\right]=\frac{1}{\alpha-\beta}\left[\begin{array}{cc}
\alpha & \beta \\
1 & 1
\end{array}\right]\left[\begin{array}{cc}
\alpha^{\mathrm{n}} & 0 \\
0 & \beta^{\mathrm{n}}
\end{array}\right]\left[\begin{array}{c}
1 \\
-1
\end{array}\right]
$$

Logo,

$$
\mathrm{F}_{\mathrm{n}}=\frac{\alpha^{\mathrm{n}}-\beta^{\mathrm{n}}}{\alpha-\beta},
$$

como queríamos.

Essa identidade é conhecida como fórmula de Binet, em homenagem ao matemático francês Jacques-Philippe-Marie Binet (1786-1856), que a descobriu em 1843. Na verdade, a fórmula foi descoberta pela primeira vez em 1718, pelo matemático francês Abraham De Moivre (1667-1754), usando funções geradoras. Daí, em 1844, o engenheiro e matemático francês Gabriel Lamé (17951870) também a descobriu, de forma independente [2, p. 79].

De todo modo, a fórmula pode ser utilizada para obter uma miríade de outras identidades, e obteremos algumas a seguir.

Corolário 2 (Lucas). Seja n um inteiro $\geq 0$. Então

$$
\mathrm{F}_{\mathrm{n}+1}^{2}+\mathrm{F}_{\mathrm{n}}^{2}=\mathrm{F}_{2 \mathrm{n}+1} \text {. }
$$

Demonstração. Utilizamos as relações $\alpha-\beta=\sqrt{5}, \alpha \beta=-1$ e as Identidades (7) e (8) para obter

$$
\begin{aligned}
\mathrm{F}_{\mathrm{n}+1}^{2}+\mathrm{F}_{\mathrm{n}}^{2} & =\frac{\left(\alpha^{\mathrm{n}+1}-\beta^{\mathrm{n}+1}\right)^{2}}{(\alpha-\beta)^{2}}+\frac{\left(\alpha^{\mathrm{n}}-\beta^{\mathrm{n}}\right)^{2}}{(\alpha-\beta)^{2}} \\
& =\frac{\left(\alpha^{2 \mathrm{n}+2}-2 \alpha^{\mathrm{n}+1} \beta^{\mathrm{n}+1}+\beta^{2 \mathrm{n}+2}\right)+\left(\alpha^{2 \mathrm{n}}-2 \alpha^{\mathrm{n}} \beta^{\mathrm{n}}+\beta^{2 \mathrm{n}}\right)}{5} \\
& =\frac{\left(\alpha^{2 \mathrm{n}+2}+\beta^{2 \mathrm{n}+2}\right)-2 \alpha^{2 \mathrm{n}} \beta^{2 \mathrm{n}}(1+\alpha \beta)+\left(\alpha^{2 \mathrm{n}}+\beta^{2 \mathrm{n}}\right)}{5} \\
& =\frac{\mathrm{L}_{2 \mathrm{n}+2}+\mathrm{L}_{2 \mathrm{n}}}{5} .
\end{aligned}
$$

Utilizando a Identidade (4), obtemos

$$
\begin{aligned}
\mathrm{L}_{2 \mathrm{n}+2}+\mathrm{L}_{2 \mathrm{n}} & =\mathrm{F}_{2 \mathrm{n}+3}+\mathrm{F}_{2 \mathrm{n}+1}+\mathrm{F}_{2 \mathrm{n}+1}+\mathrm{F}_{2 \mathrm{n}-1} \\
& =\mathrm{F}_{2 \mathrm{n}+2}+\mathrm{F}_{2 \mathrm{n}+1}+2 \mathrm{~F}_{2 \mathrm{n}+1}+\mathrm{F}_{2 \mathrm{n}-1} \\
& =\mathrm{F}_{2 \mathrm{n}+1}+\mathrm{F}_{2 \mathrm{n}}+3 \mathrm{~F}_{2 \mathrm{n}+1}+\mathrm{F}_{2 \mathrm{n}-1} \\
& =4 \mathrm{~F}_{2 \mathrm{n}+1}+\mathrm{F}_{2 \mathrm{n}}+\mathrm{F}_{2 \mathrm{n}-1} \\
& =5 \mathrm{~F}_{2 \mathrm{n}+1} .
\end{aligned}
$$

Logo,

$$
\mathrm{F}_{\mathrm{n}+1}^{2}+\mathrm{F}_{\mathrm{n}}^{2}=\frac{5 \mathrm{~F}_{2 \mathrm{n}+1}}{5}=\mathrm{F}_{2 \mathrm{n}+1}
$$

como queríamos demonstrar. 
Existem apenas dois números de Fibonacci distintos que são quadrados perfeitos: 1 e 144. Consequentemente, a Identidade (10), mostrada por É. Lucas, em 1876, tem uma bela interpretação geométrica: não há números de Fibonacci consecutivos que possam ser comprimentos de um triângulo retângulo.

Entretanto, o triângulo $\mathrm{ABC}$, com $\mathrm{AC}=\mathrm{F}_{\mathrm{k}} \mathrm{F}_{\mathrm{k}+3}, \mathrm{BC}=2 \mathrm{~F}_{\mathrm{k}+1} \mathrm{~F}_{\mathrm{k}+2}$ e $\mathrm{AB}=\mathrm{F}_{2 \mathrm{k}+3}$, onde $\mathrm{k}$ é um inteiro $\geq 0$, é retângulo em C, conforme estabelecido por C. W. Raine em 1948 [2, p. 79].

De fato,

$$
\begin{aligned}
\mathrm{AC}^{2}+\mathrm{BC}^{2} & =\left(\mathrm{F}_{\mathrm{k}} \mathrm{F}_{\mathrm{k}+3}\right)^{2}+\left(2 \mathrm{~F}_{\mathrm{k}+1} \mathrm{~F}_{\mathrm{k}+2}\right)^{2} \\
& =\left(\mathrm{F}_{\mathrm{k}+2}-\mathrm{F}_{\mathrm{k}+1}\right)^{2}\left(\mathrm{~F}_{\mathrm{k}+2}+\mathrm{F}_{\mathrm{k}+1}\right)^{2}+4 \mathrm{~F}_{\mathrm{k}+1}^{2} \mathrm{~F}_{\mathrm{k}+2}^{2} \\
& =\left(\mathrm{F}_{\mathrm{k}+2}^{2}-\mathrm{F}_{\mathrm{k}+1}^{2}\right)^{2}+4 \mathrm{~F}_{\mathrm{k}+1}^{2} \mathrm{~F}_{\mathrm{k}+2}^{2} \\
& =\mathrm{F}_{\mathrm{k}+2}^{4}-2 \mathrm{~F}_{\mathrm{k}+1}^{2} \mathrm{~F}_{\mathrm{k}+2}^{2}+\mathrm{F}_{\mathrm{k}+1}^{4}+4 \mathrm{~F}_{\mathrm{k}+1}^{2} \mathrm{~F}_{\mathrm{k}+2}^{2} \\
& =\mathrm{F}_{\mathrm{k}+2}^{4}+2 \mathrm{~F}_{\mathrm{k}+1}^{2} \mathrm{~F}_{\mathrm{k}+2}^{2}+\mathrm{F}_{\mathrm{k}+1}^{4} \\
& =\left(\mathrm{F}_{\mathrm{k}+2}^{2}+\mathrm{F}_{\mathrm{k}+1}^{2}\right)^{2} .
\end{aligned}
$$

Utilizando o Corolário 2, $\mathrm{AC}^{2}+\mathrm{BC}^{2}=\mathrm{F}_{2 \mathrm{k}+3}^{2}=\mathrm{AB}^{2}$ e o teorema de Pitágoras nos garante que $\triangle \mathrm{ABC}$ é retângulo em $\mathrm{C}$.

Corolário 3. Seja n um inteiro $\geq 0$. Então,

$$
\mathrm{F}_{\mathrm{n}} \mathrm{L}_{\mathrm{n}}=\mathrm{F}_{2 \mathrm{n}} .
$$

Demonstração. Valemo-nos dos Identidades (7) e (8):

$$
\mathrm{F}_{\mathrm{n}} \mathrm{L}_{\mathrm{n}}=\frac{\alpha^{\mathrm{n}}-\beta^{\mathrm{n}}}{\alpha-\beta}\left(\alpha^{\mathrm{n}}+\beta^{\mathrm{n}}\right)=\frac{\alpha^{2 \mathrm{n}}-\beta^{2 \mathrm{n}}}{\alpha-\beta}=\mathrm{F}_{2 \mathrm{n}} \text {. }
$$

A Identidade (11) implica que, quando $\mathrm{n} \geq 3$, todo número de Fibonacci da forma $\mathrm{F}_{2 \mathrm{n}}$ (com índice par) é composto, isto é, possui fatores não triviais.

Aplicando-se o Teorema 5 pode-se obter uma generalização do Teorema 4:

$$
\mathrm{F}_{\mathrm{n}+\mathrm{k}} \mathrm{F}_{\mathrm{n}-\mathrm{k}}-\mathrm{F}_{\mathrm{n}}^{2}=(-1)^{\mathrm{n}+\mathrm{k}+1} \mathrm{~F}_{\mathrm{k}}^{2},
$$

onde $\mathrm{n} \geq \mathrm{k}$, demonstrada pelo matemático belga Eugène Charles Catalan (1814-1894) em 1879 [2, pp. 82-83].

\subsection{O método de Newton e a razão áurea}

No início desta seção, vimos que as frações contínuas

$$
1+\frac{1}{1+\frac{1}{1+\frac{1}{1+\cdots}}}
$$


convergem para o número $\alpha$.

Podemos também representar a razão áurea em termos de radicais. Com efeito, fazendo

$$
x=\sqrt{1+\sqrt{1+\sqrt{1+\ldots}}}
$$

obtemos

$$
x^{2}=1+\sqrt{1+\sqrt{1+\sqrt{1+\ldots}}},
$$

ou seja, $\mathrm{x}^{2}=1+\mathrm{x}$. As raízes dessa nossa já conhecida equação são $\alpha$ e $\beta$, donde, porque positivo, $\mathrm{x}=\alpha$.

As frações contínuas e os radicais acima fornecem métodos para a aproximação da razão áurea. Sua convergência, no entanto, é bastante lenta, como poderá verificar o leitor.

Em 1999, J. W. Roche, do Colégio La Salle de Wyndmoor, Pensilvânia, estimou a razão áurea utilizando o método de Newton para aproximar um dos zeros da função polinomial

$$
f(x)=x^{2}-x-1
$$

No processo, ele encontrou uma relação entre as várias aproximações e os números de Fibonacci [2, pp. 245-246].

Usando o valor inicial $\mathrm{x}_{1}=2$ e a fórmula recursiva

$$
x_{n+1}=x_{n}-\frac{f\left(x_{n}\right)}{f^{\prime}\left(x_{n}\right)},
$$

em que $f^{\prime}\left(x_{n}\right)$ é a derivada de $f$ no ponto $x_{n}$, ele encontrou as três seguintes aproximações:

$$
\mathrm{x}_{2}=\frac{5}{3}, \quad \mathrm{x}_{3}=\frac{34}{21} \quad \text { e } \quad \mathrm{x}_{4}=\frac{1597}{987} .
$$

Percebendo que todas elas são razões de números de Fibonacci consecutivos, conjecturou:

$$
\mathrm{x}_{\mathrm{n}}=\frac{\mathrm{F}_{2^{\mathrm{n}}+1}}{\mathrm{~F}_{2^{\mathrm{n}}}},
$$

onde $\mathrm{n} \geq 1$.

A validade da conjectura de Roche pode ser demonstrada aplicando-se os Corolários 2 e 3 e utilizando o princípio de indução finita.

De fato, uma vez que $\mathrm{F}_{3} / \mathrm{F}_{2}=2 / 1=\mathrm{x}_{1}$, a afirmação é válida para $\mathrm{n}=1$. Assumimos então que a fórmula funcione para um inteiro $\mathrm{n}>1$ arbitrário, isto é, que $\mathrm{x}_{\mathrm{n}}=\mathrm{F}_{\mathrm{m}+1} / \mathrm{F}_{\mathrm{m}}$, onde $\mathrm{m}=2^{\mathrm{n}}$. Porque $\mathrm{f}^{\prime}(\mathrm{x})=2 \mathrm{x}-1$

$$
\begin{aligned}
x_{n+1} & =x_{n}-\frac{x_{n}^{2}-x_{n}-1}{2 x_{n}-1}=\frac{2 x_{n}^{2}-x_{n}-x_{n}^{2}+x_{n}+1}{2 x_{n}-1} \\
& =\frac{x_{n}^{2}+1}{2 x_{n}-1}=\frac{F_{m+1}^{2} / F_{m}^{2}+1}{2 F_{m+1} / F_{m}-1}=\frac{F_{m+1}^{2}+F_{m}^{2}}{F_{m}\left(2 F_{m+1}-F_{m}\right)} \\
& =\frac{F_{2 m+1}}{F_{m}\left(F_{m+1}+F_{m-1}\right)}=\frac{F_{2 m+1}}{F_{m} L_{m}}=\frac{F_{2 m+1}}{F_{2 m}}
\end{aligned}
$$


e a fórmula funciona para $n+1$. Assim, pelo princípio de indução finita, a afirmação é verdadeira para todo inteiro $n \geq 1$.

Como $\lim _{\mathrm{m} \rightarrow \infty} \mathrm{F}_{\mathrm{m}+1} / \mathrm{F}_{\mathrm{m}}=\alpha$, segue que a sequência de aproximações $\left\{\mathrm{x}_{\mathrm{n}}\right\}$ converge para o número de ouro $\alpha$, como era esperado.

\section{Considerações finais}

Vimos que o número $\alpha=(\sqrt{5}+1) / 2$ emerge da divisão de um segmento na qual a razão entre maior e menor partes é igual à razão entre o segmento todo e a maior parte. Aprendemos duas maneiras de dividir, com régua e compasso, um segmento na razão áurea. Definimos triângulo áureo e vimos que ele aparece na construção tanto do decágono quanto do pentágono regulares. Mostramos como construir um retângulo áureo, bem como obter sucessivos retângulos áureos recíprocos. A partir da união de quadrantes circulares, vimos surgir nesses retângulos áureos uma curva que aproxima a espiral logarítmica.

Conhecemos um pouco da história do matemático Leonardo Pisano, que ajudou a popularizar os algarismos hindu-arábicos por meio de seu Liber Abaci. Nesse livro, Fibonacci, como ficou conhecido, apresentou também o problema da reprodução de coelhos, em cuja solução figuram os números $1,1,2,3,5,8$ etc. A regra para sua célebre sequência, como ele não poderia ter deixado de notar, é que cada termo, $F_{n}$, é obtido da soma dos dois termos predecessores, $F_{n-1} e$ $\mathrm{F}_{\mathrm{n}-2}$. Curiosamente, a razão $\mathrm{F}_{\mathrm{n}} / \mathrm{F}_{\mathrm{n}-1}$ é tanto mais próxima do número $\alpha$ quanto maior o inteiro n. Demonstramos essa afirmação. Apresentamos duas demonstrações para a fórmula de Binet e vimos como obter algumas identidades a partir dela. Vimos também como o princípio de indução pode ser utilizado para demonstrar a fórmula de Cassini e a convergência de uma sequência de aproximações para a raiz positiva do polinômio $\mathrm{x}^{2}-\mathrm{x}-1$.

Dessa forma, além de aprender diversos conceitos da geometria, álgebra linear, análise real e teoria dos números, pudemos apreciar algumas das contribuições de grandes matemáticos para a história e evolução desta ciência. A matemática, se bem que exata, é feita do esforço e dedicação diários daqueles que, abnegada e apaixonadamente, procuram desvendar-lhe os mistérios.

\section{Agradecimentos}

Agradeço à Juliana, que quis saber mais sobre a sequência de Fibonacci e a razão áurea e, assim, inspirou a criação deste artigo.

Agradeço à minha namorada, Aline, sem cujos carinhos e cuidados, não apenas este artigo não seria possível, a vida teria menos cor, menos sabor, menos graça.

\section{Referências}

[1] ARCHIBALD, R. C. Golden section. The American Mathematical Monthly, Taylor \& Francis, Ltd. on behalf of the Mathematical Association of America, v. 25, n. 5, p. 232-238, 1918. Disponível em: https://www.jstor.org/stable/2972652.

[2] KOSHY, T. Fibonacci and Lucas Numbers with Applications. $1^{\mathrm{a}}$ ed. New York: John Wiley \& Sons, Inc., 2001. (Pure and applied mathematics).

[3] NIEMEYER, J. A simple construction of the golden section. Forum Geometricorum, v. 11, p. 53, 2011. Disponível em: http://forumgeom.fau.edu/FG2011volume11/FG201105.pdf. 
[4] BATAILLE, M. Another simple construction of the golden section. Forum Geometricorum, v. 11, p. 55, 2011. Disponível em: http://forumgeom.fau.edu/FG2011volume11/FG201106.pdf.

[5] MUNIZ NETO, A. C. Tópicos de Matemática Elementar. 2ª ed. Rio de Janeiro: SBM, 2013. v. 2, Geometria Euclidiana. (Coleção do Professor de Matemática, v. 2, Geometria Euclidiana).

[6] COXETER, H. S. M. Introduction to Geometry. $2^{\mathrm{a}}$ ed. New York: John Wiley \& Sons, Inc., 1989. (New Mathematical Library).

[7] ANDREWS, G. E. Number Theory. $1^{\mathrm{a}}$ ed. West Washington Square, Philadelphia: W. B. Sanders Company, 1971.

[8] LIMA, E. L. et al. A Matemática do Ensino Médio. $7^{\mathrm{a}}$ ed. Rio de Janeiro: SBM, 2016. v. 2. (Coleção do Professor de Matemática, v. 2).

[9] STRANG, G. Introduction to Linear Algebra. $4^{\mathrm{a}}$ ed. New York: Wellesley-Cambridge Press, 2009.

Yuri Teles Moura

Brasília, Distrito Federal

$<$ yuriorim@gmail.com>

Recebido: 19/09/2020

Publicado: 15/04/2021 